\title{
PERNIL DE CORDEIRO MATURADO SOB REFRIGERAÇÃO E ACONDICIONADO EM DIFERENTES SISTEMAS DE EMBALAGEM COM ATMOSFERA MODIFICADA
}

\author{
F. R. DANTAS ${ }^{1,2}$, L.S. SOARES ${ }^{1}$, J.C.C. PETRUS ${ }^{1}$, M.S. MADRUGA ${ }^{3}$, A.R.MONTEIRO ${ }^{1}$, \\ ${ }^{1}$ Universidade Federal de Santa Catarina, Departamento de Engenharia Química e Engenharia de \\ Alimentos, Pós-graduação em Engenharia de Alimentos \\ ${ }^{2}$ Instituto Federal de Educação, Ciência e Tecnologia do Sertão Pernambucano, Campus Floresta \\ ${ }^{3}$ Universidade Federal da Paraíba, Departamento de Ciência e Tecnologia, Pós-graduação em \\ Engenharia de Alimentos \\ E-mail para contato: fabiana.dantas@ifsertao-pe.edu.br
}

\begin{abstract}
RESUMO - Este trabalho teve como objetivo estudar a influencia de diferentes sistemas de acondicionamento na vida útil de pernil de cordeiro maturado sob refrigeração. Os produtos foram maturados a $3^{\circ} \mathrm{C}$ sob as seguintes condições: vácuo, em atmosferas modificadas (contendo $70 \% \mathrm{CO}_{2} / 30 \%$ de $\mathrm{N}_{2}$ e $100 \%$ de $\mathrm{CO}_{2}$ ), e em ar atmosférico (controle) por 28 dias. Foram realizadas análises de atividade água, cor, pH, composição centesimal, perda de água e análises microbiológicas - contagem de coliformes totais e termotolerantes, psicrófilos, psicrotróficos, mesófilos, bactérias lácticas e presença de Salmonella. A contagem microbiana durante 14 dias foi inferior para as amostras maturadas a vácuo e sob atmosfera modificada, enquanto sob ar atmosférico foi quatro vezes superior à contagem inicial. Entre as atmosferas modificadas, as amostras maturadas em $100 \%$ de $\mathrm{CO}_{2}$ apresentaram maior vida útil.
\end{abstract}

\section{INTRODUÇÃO}

A produção de carne ovina no Brasil está em ascensão e sua comercialização é predominantemente sob a forma congelada e, como consequência, tem atraído pouco os consumidores, que desejam produtos que ofereçam segurança e praticidade. Deste modo, estudos vêm sendo realizados com o objetivo de aumentar a vida de prateleira destes produtos, além de manter e/ou melhorar as características sensoriais relacionadas com sabor, cor e textura (MADRUGA et al., 2005; MONTE et al, 2012).

A maturação é utilizada visando melhorar os atributos sensoriais da carne, através da ação de enzimas proteolíticas que hidrolisam parcialmente as miofibrilas, resultando no aumento de sua maciez e suculência. Produtos cárneos embalados sob atmosfera modificada (AM) têm seu sabor e maciez intensificados. Fernandes et al. (2014) verificaram que a aplicação de $\mathrm{CO}_{2}$ durante a maturação e armazenamento de carne ovina com atmosfera modificada, não altera suas características físico-químicas.

Neste sentido, diversas pesquisas têm mostrado resultados promissores referentes ao aumento da vida útil de carne ovina levando à obtenção de produtos com características sensoriais e microbiológicas desejáveis, após o período de maturação com uso de atmosfera modificada (FERNANDES et al, 2014). 
O objetivo deste trabalho foi avaliar a influência de diferentes sistemas de acondicionamento na vida de útil de pernil de cordeiro maturado sob refrigeração.

\section{MATERIAL E MÉTODOS}

Pernis desossados obtidos de cordeiro da raça Ile de France, com média de 1 ano de idade, recém abatidos, foram adquiridos em frigorífico com inspeção estadual, na grande Florianópolis/SC. Foram transportados em recipiente hermético isotérmico com gelo para o Laboratório de Propriedades Físicas de Alimentos - UFSC, onde foram fracionados em fatias de $300 \pm 50 \mathrm{~g}$. Estas amostras foram maturadas em embalagens flexíveis de alta barreira compostas de polietileno e poliamida. Os experimentos seguiram um planejamento fatorial 4 $\mathrm{x} 4$, tipo de acondicionamento (controle $-\mathrm{t}_{1}$; vácuo $-\mathrm{t}_{2} ; 70 \%$ de $\mathrm{CO}_{2}+30 \%$ de $\mathrm{N}_{2}-\mathrm{t}_{3}, 100 \%$ de $\left.\mathrm{CO}_{2}-\mathrm{t}_{4}\right)$ e tempo de maturação $\left(7,14,21\right.$ e 28 dias) a $3^{\circ} \mathrm{C}$.

As misturas de gás utilizadas foram $70 \% \mathrm{CO}_{2}+30 \%$ de $\mathrm{N}_{2}$ (Conservare 62 Air Liquide) e $100 \%$ de $\mathrm{CO}_{2}$ (ALIGAL White Martins). O envase dos produtos foi realizado em seladora com injeção de gás, com tempo de ajuste no momento da injeção de $10 \mathrm{~s}$ de vácuo e $9 \mathrm{~s}$ da mistura gasosa. Em seguida as embalagens foram seladas. Os gases foram monitorados mediante a leitura da concentração no interior das embalagens utilizando-se analisador de gases (CheckMate II, PBI Dansensor).

A vida de prateleira dos produtos maturados foi determinada por meio dos seguintes parâmetros:

- Composição centesimal: As determinações de umidade, cinzas, proteínas e lipídeos foram realizadas de acordo com as normas analíticas da AOAC (2000).

- Medida da cor: Foi realizada segundo metodologia descrita por Abularach; Rocha; Felício (1998), utilizando colorímetro digital, no espaço colorimétrico CIELAB, definido por $\mathrm{L}^{*}, \mathrm{a}^{*}, \mathrm{~b}^{*}$. A coordenada $\mathrm{L}^{*}$ corresponde ao teor de luminosidade (0 a 100 - preto ao branco), $a^{*}$ à cromaticidade verde $(-a) / v e r m e l h o(+a)$ e b*à azul(-b)/amarelo(+b). As amostras foram descongeladas a $4^{\circ} \mathrm{C}$ por 24 horas e expostas ao ar atmosférico por um período de 45 minutos para proceder a leitura da cor em três pontos distintos das amostras.

- $p H$ : Utilizou-se potenciômetro digital de penetração diretamente nas amostras antes e após cada condição de maturação A medida foi realizada em três pontos de cada amostra, próximo às extremidades esquerda e direita e no centro.

- Atividade de água (aw): Foi determinada pelo método direto 978.18, descrito pela AOAC (2000), utilizando-se de higrômetro (Aqualab, da Decagon Devices).

- Capacidade de retenção de água (CRA): A capacidade de retenção de água foi determinada utilizando-se método modificado de centrifugação, conforme descrito por Desmond et al. (2000).

- Perda de Água por Cocção (PAC): foi realizada de acordo com metodologia adaptada a partir de AMSA (1995). 
- Análises microbiológicas: Pesquisou-se a incidência de Coliformes totais e termotolerantes, Salmonella, psicrófilos, psicrotróficos, mesófilos e bactérias ácido lácticas (BAL) nas amostras frescas (tempo zero). As amostras maturadas foram amostradas a cada 7 dias, de forma aleatória aos 7, 14, 21 e 28 dias, e enviadas para análise laboratorial de psicrófilos. Todas as análises foram realizadas conforme ISO 7937 (2007).

Os resultados obtidos foram avaliados por análise de variância (ANOVA) e as médias foram comparadas através do teste de Tukey, em nível de 5\% de significância.

\section{RESULTADOS E DISCUSSÃO}

\subsection{Composição gasosa nas embalagens sob AM}

Como esperado em produtos cárneos, na Figura 1 observou-se uma redução da concentração de $\mathrm{CO}_{2}$ no volume livre das embalagens. Atribui-se esta redução à dissolução do gás no produto (FERNANDES et al., 2014), que ocorre nas primeiras horas após o envase. Tendo em vista que a composição da AM é dinâmica e sofre influência do metabolismo microbiano, da absorção do gás no produto e da permeabilidade do material da embalagem (JAKOBSEN \& BERTELSEN 2004). A presença de $\mathrm{O}_{2}$ nas embalagens foi praticamente zero ao longo da maturação e as embalagens utilizadas foram eficazes na conservação dos gases em seu interior.

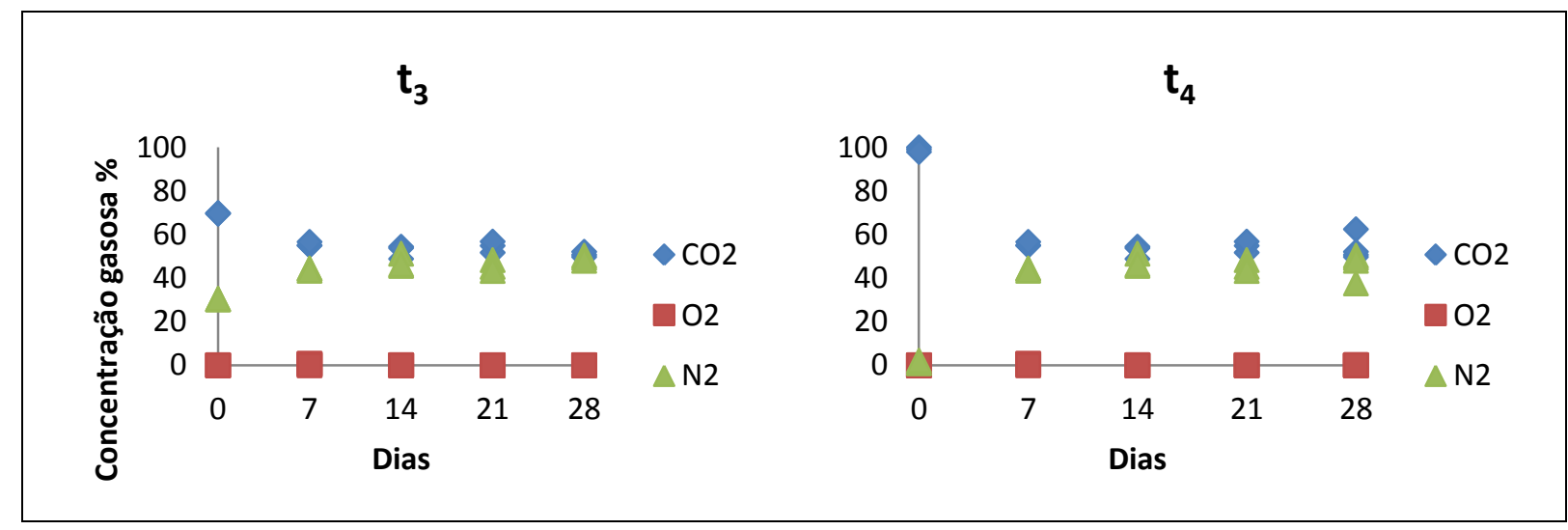

Figura 1. Composição gasosa nas embalagens durante a maturação nos tratamentos sob AM $70 \%$ de $\mathrm{CO}_{2}+30 \%$ de $\mathrm{N}_{2}\left(\mathrm{t}_{3}\right)$ e $100 \%$ de $\mathrm{CO}_{2}\left(\mathrm{t}_{4}\right)$ com a $3^{\circ} \mathrm{C}$.

\subsection{Caracterização físico-química}

O teor de umidade, proteína, resíduo mineral e lipídeos foi 72,60 $\pm 0,46,19,01 \pm 0,31$, $0,94 \pm 0,017,8,34 \pm 0,15 \mathrm{~g} / 100 \mathrm{~g}$, respectivamente. Estes valores são compatíveis com relatos da literatura (JARDIM et al., 2007; FERNANDES et al., 2012), a exceção do teor de lipídeos que foi elevado quando comparado ao teor de outros cortes cárneos. Isto se deve a composição própria do pernil, tendo em vista a composição muscular variada, tecido conjuntivo e gordura entremeada na fibra muscular. 
Os parâmetros de cor $\left(\mathrm{L}^{*}, \mathrm{a}^{*}, \mathrm{~b}^{*}\right)$ (Tabela 1) não apresentaram variação $(\mathrm{p}>0,05)$ ao analisar as amostras com e sem embalagem. Informação relevante, considerando-se que a cor do produto embalado é um parâmetro importante para o consumidor no momento da compra.

Considerando-se o tempo de maturação, a intensidade de luminosidade $L^{*}$ diferiu $(p<0,05)$ entre tratamentos nas amostras com embalagem aos 7 dias. Enquanto que aos 14 dias, diferiu $(\mathrm{p}<0,05)$ com e sem embalagens aos 14 dias, quando analisada a influencia dos sistemas de acondicionamento. Estas diferenças podem ser atribuídas à presença de $\mathrm{CO}_{2}$ na composição gasosa nas embalagens.

Não houve perda de coloração quanto à luminosidade durante o período de maturação das amostras $(\mathrm{p}>0,05)$. Esse fenômeno pode estar relacionado à manutenção do $\mathrm{pH}$ no período, diferentemente do esperado, tendo em vista que a maturação promove o escurecimento e perda da luminosidade pela ação da oximioglobina (JAKOBSEN \& BERTELSEN, 2004). Acredita-se que a rancificação e/ou oxidação lipídica nas amostras foi mínima, bem como a desnaturação das proteínas, já que não houve aumento dos valores médios de $\mathrm{L}^{*}$, fator que aumenta a dispersão da luz (MANO et al., 2002).

Tabela 1. Medição de cor das amostras maturadas sob vácuo, atmosfera modificada com $70 \%$ $\mathrm{CO}_{2}$, atmosfera modificada com $100 \% \mathrm{CO}_{2}$ e em ar atmosférico aos $7,14,21$ e 28 dias a $3^{\circ} \mathrm{C}$

\begin{tabular}{|c|c|c|c|c|c|c|c|c|c|}
\hline \multirow{3}{*}{ Tempo } & \multirow{3}{*}{ Parâmetros } & \multicolumn{8}{|c|}{ Tratamentos } \\
\hline & & \multicolumn{2}{|c|}{ Controle } & \multicolumn{2}{|c|}{ Vácuo } & \multicolumn{2}{|c|}{ AM 70\% } & \multicolumn{2}{|c|}{ AM $100 \%$} \\
\hline & & c/e & s/e & c/e & s/e & c/e & s/e & c/e & s/e \\
\hline \multirow{3}{*}{7} & $a^{*}$ & 10,87 & 12,00 & 12,21 & 13,28 & 11,28 & 13,42 & 11,13 & 11,82 \\
\hline & $\mathrm{b}^{*}$ & 9,82 & 10,03 & 9,13 & 10,16 & 9,38 & 10,57 & 10,71 & 10,10 \\
\hline & L* & $39,69^{\mathrm{a}}$ & 42,09 & $30,89^{a}$ & 39,57 & $36,9^{\mathrm{a}}$ & 37,24 & $41,05^{b}$ & 42,76 \\
\hline \multirow{3}{*}{14} & $a^{*}$ & 11,24 & 13,28 & 10,66 & 12,56 & 10,34 & 11,34 & 10,70 & 11,33 \\
\hline & $b^{*}$ & 11,43 & 12,37 & 9,56 & 10,35 & 9,04 & 9,04 & 10,70 & 10,62 \\
\hline & $\mathrm{L}^{*}$ & $40,94^{\mathrm{a}}$ & $44,34^{\mathrm{a}}$ & $39,79^{\mathrm{ab}}$ & $41,84^{\mathrm{ab}}$ & $39,66^{\mathrm{b}}$ & $39,23^{\mathrm{b}}$ & $38,9^{\mathrm{a}}$ & $43,82^{\mathrm{a}}$ \\
\hline \multirow{3}{*}{21} & $a^{*}$ & 10,81 & - & 10,39 & 11,36 & 10,12 & 11,11 & 10,03 & 11,76 \\
\hline & $b^{*}$ & 11,48 & - & 9,31 & 9,89 & 9,34 & 9,79 & 9,02 & 9,34 \\
\hline & $\mathrm{L}^{*}$ & 42,58 & - & 38,54 & 43,29 & 36,91 & 42,22 & 39,48 & 42,69 \\
\hline \multirow{3}{*}{28} & $a^{*}$ & 8,5 & - & 8,88 & - & 8,02 & - & 9,23 & 11,82 \\
\hline & $b^{*}$ & 8,65 & - & 9,57 & - & 8,01 & - & 8,86 & 10,10 \\
\hline & $\mathrm{L}^{*}$ & 40,79 & - & 41,77 & - & 40,91 & - & 40,48 & 42,76 \\
\hline
\end{tabular}

c/e- com embalagem; s/e- sem embalagem, medida feita 45 min após a abertura do produto em triplicata; a* cromaticidade verde (-)/vermelho $(+), b^{*}$ (azul(-)/amarelo $(+)$ e $\mathrm{L}^{*}$ (luminosidade). Valores seguidos por letras distintas nas linhas diferem entre si pelo teste Tukey $(\mathrm{p}<0,05)$. Dentro de um mesmo tempo, valores seguidos de letras minúsculas iguais não diferem entre si pelo teste Tukey. Ausência de letra quando ( $p>0,05)$

Em $\mathrm{AM}$ de $70 \%$ de $\mathrm{CO}_{2}+30 \% \mathrm{~N}$, a intensidade de $\mathrm{L} *$ foi equivalente às amostras maturadas sob vácuo, apresentando diferença significativa quando contrastado ao grupo controle e com $100 \%$ de $\mathrm{CO}_{2}$ aos 14 dias, não havendo diferença significativa entre as amostras embaladas sob vácuo e AM aos 21 dias. Fernandes et al. (2014) estudaram o lombo 
de cordeiro estocado $\left(4^{\circ} \mathrm{C}\right)$ por 21 dias com vácuo, $75 \%$ de $\mathrm{O}_{2}+25 \%$ de $\mathrm{CO}_{2}$ e $100 \%$ de $\mathrm{CO}_{2}$ e encontraram valores médios de $\mathrm{L}^{*} 36,95 \pm 1,05,38,47 \pm 1,05$ e 36,32 $\pm 1,05$, respectivamente, inferiores aos obtidos neste estudo em relação ao vácuo $(43,29)$ e $100 \%$ de $\mathrm{CO}_{2}(42,69)$.

A maturação e o tipo de acondicionamento não afetaram $(p>0,05)$ os teores de $a^{*}$ e $b^{*}$. No entanto, verifica-se uma tendência de cor verde (-a*) no grupo controle a partir dos 14 dias e nas amostras sob vácuo e AM após 21 dias. Quando sob AM de $100 \% \mathrm{CO}_{2}$, houve a manutenção da coloração avermelhada brilhante durante todo o período de maturação.

O teor a* das amostras com $100 \%$ de $\mathrm{CO}_{2}$ aos 21 dias $(11,76)$ mostra a eficácia do uso desta atmosfera na manutenção da coloração da carne, tendo em vista que descoloração superficial ocorre quando se identifica valores mais elevados de $a^{*}(>15)$. Verifica-se diferença entre os cortes cárneos (pernil e lombo) maturados sob mesma atmosfera, considerando que Fernandes et al. (2014) encontraram teor de $1^{*}(13,01 \pm 0,54)$ mais elevado em lombo de cordeiro maturado $\left(4^{\circ} \mathrm{C}\right)$.

Observa-se uma pequena redução nas médias de $b^{*}$, que pode ser devida à baixa quantidade de $\mathrm{O}_{2}$ presente no sistema. Em pesquisas com AM, contendo diferentes níveis de $\mathrm{O}_{2}$, Vergara e Galego (2001) e Fernandes et al. (2014) encontraram valores de b* crescentes, pela ação da oximioglobina .

Tabela 2. Valores médios de pH, atividade água, capacidade de retenção de água e perda de água por cocção da carne de cordeiro fresca (AF) e carne de cordeiro maturadas em sistemas de vácuo, atmosfera modificada com $70 \% \mathrm{CO}_{2}$ e com $100 \% \mathrm{CO}_{2}$, e grupo controle, aos 7,14 , 21 dias a $3^{\circ} \mathrm{C}$

\begin{tabular}{|c|c|c|c|c|c|}
\hline Tratamento & $\begin{array}{c}\text { Tempo } \\
\text { (dias) }\end{array}$ & pH & aw & CRA & PAC \\
\hline AF & 0 & 5,81 & 0,995 & 35,33 & 34,54 \\
\hline \multirow{2}{*}{ Controle } & 7 & $5,60^{\mathrm{a} A}$ & $0,992^{\mathrm{aA}}$ & $33,35^{\mathrm{aA}}$ & $48,99^{\mathrm{aA}}$ \\
\hline & 14 & $5,72^{\mathrm{aA}}$ & $0,992^{\mathrm{aA}}$ & $24,60^{\mathrm{aA}}$ & $44,52^{\mathrm{aA}}$ \\
\hline \multirow{3}{*}{ Vácuo } & 7 & $5,55^{\mathrm{aA}}$ & $0,991^{\mathrm{aA}}$ & $35,89^{\mathrm{bA}}$ & $48,92^{\mathrm{bA}}$ \\
\hline & 14 & $5,61^{\mathrm{aA}}$ & $0,994^{\mathrm{aA}}$ & $26,59^{\mathrm{abA}}$ & $46,91^{\mathrm{bA}}$ \\
\hline & 21 & $5,45^{\mathrm{aA}}$ & $0,993^{\mathrm{aB}}$ & $26,36^{\mathrm{aA}}$ & $36,43^{\mathrm{aA}}$ \\
\hline \multirow{3}{*}{$\begin{array}{c}\text { ATM } \\
70 \% \mathrm{CO} 2\end{array}$} & 7 & $5,61^{\mathrm{a} A}$ & $0,992^{\mathrm{aA}}$ & $34,81^{\mathrm{aA}}$ & $44,92^{\mathrm{aA}}$ \\
\hline & 14 & $5,65^{\mathrm{aA}}$ & $0,991^{\mathrm{aA}}$ & $26,78^{\mathrm{abA}}$ & $48,44^{\mathrm{aA}}$ \\
\hline & 21 & $5,47^{\mathrm{aA}}$ & $0,990^{\mathrm{aA}}$ & $26,22^{\mathrm{bA}}$ & $56,82^{\mathrm{aB}}$ \\
\hline \multirow{3}{*}{$\begin{array}{c}\text { ATM } \\
100 \% \mathrm{CO} 2\end{array}$} & 7 & $5,59^{\mathrm{a} A}$ & $0,993^{\mathrm{a} A}$ & $34,02^{\mathrm{bA}}$ & $40,96^{\mathrm{aA}}$ \\
\hline & 14 & $5,56^{\mathrm{aA}}$ & $0,991^{\mathrm{aA}}$ & $27,03^{\mathrm{aA}}$ & $39,97^{\mathrm{aA}}$ \\
\hline & 21 & $5,61^{\mathrm{aA}}$ & $0,990^{\mathrm{aA}}$ & $21,72^{\mathrm{aA}}$ & $41,00^{\mathrm{aA}}$ \\
\hline
\end{tabular}

Letras minúsculas e maiúsculas diferentes diferem entre si pelo teste Tukey $(\mathrm{p}<0,05)$, para tratamentos e tempo, respectivamente.

Os valores médios de $\mathrm{pH}$ e aw, expressos na Tabela 2, não sofreram variações quanto à interação entre o tipo de acondicionamento e o tempo de maturação. No entanto, quando 
observada a influencia do tempo na aw aos 21 dias, verifica-se diferença $(p<0,05)$ do tratamento a vácuo em relação às demais atmosferas utilizadas.

Os valores de $\mathrm{pH}$ encontrados neste estudo estão de acordo com a faixa considerada normal $(5,5$ - 5,8) para carne de cordeiro e são semelhantes aos obtidos por Linares, Bórnez e Vergara (2008) e Bórnez, Linares e Vergara (2010) em Longissimus dorsi de cordeiro maturado. Este $\mathrm{pH}$ é ligeiramente mais elevado do que aqueles encontrados em outras carnes, como a de bovino Este fator pode favorecer o crescimento microbiano, resultando em uma menor durabilidade da carne (TERLOUW, 2005). Houve uma pequena redução no pH ao longo do tempo, provavelmente devido à dissolução de $\mathrm{CO}_{2}$ no músculo, com consequente formação de ácido carbônico (JAKOBSEN \& BERTELSEN, 2004). De acordo com Lawrie e Ledward (2006), quando não ocorre esta redução do pH, este fenômeno pode ser devido à depleção da reserva de glicogênio no momento do abate.

A CRA não variou significativamente $(\mathrm{p}>0,05)$ entre os tratamentos, mas houve variação $(\mathrm{p}<0,05)$, entre os períodos de armazenamento. A CRA diminuiu com a utilização da $\mathrm{AM}$, sendo mais pronunciada no tratamento com $100 \%$ de $\mathrm{CO}_{2}$. A perda de água por cocção variou significativamente, principalmente quando observadas as diferenças entre os tratamentos. A proporção de gases utilizada não influenciou na CRA. Entretanto, houve variação mais perceptível no tratamento sob vácuo.

\subsection{Análises microbiológicas}

A contagem inicial de microrganismos evidenciou ausência de Salmonella, e para Coliformes termotolerantes, Coliformes totais, Psicrotróficos, Psicrófilos, Mesófilos e BAL foi de $>1,0 ; 3,9 ; 3,5 ; 5,7 ; 5,4$ e $3,8 \mathrm{Log}$ (UFC/g), respectivamente. As contagens de microrganismos foram inferiores a $10^{2} \mathrm{UFC} / \mathrm{g}$, atendendo à legislação brasileira vigente. Contribuiu para este resultado, a procedência das peças de carne que foram adquiridas diretamente em abatedouro que atende às rigorosas condições de higiene e o manuseio em laboratório, também com medidas higiênicas apropriadas. Diante das baixas contagens de microrganismos totais, coliformes e ausência de Salmonella, optou-se por verificar apenas a presença dos psicrófilos que se desenvolvem numa faixa ótima de temperatura entre 0 e $7^{\circ} \mathrm{C}$.

Houve diferenças $(\mathrm{p}<0,05)$ entre os tratamentos em função do tempo quanto à carga microbiana (Figura 2). Para os tratamentos estudados o acondicionamento a vácuo e AM, contendo $70 \%$ de $\mathrm{CO}_{2} / 30 \% \mathrm{~N}_{2}$, apresentaram o mesmo tempo de vida de prateleira de 14 dias, quanto à contagem microbiológica e parâmetros físico-químicos. O tratamento com AM contendo $100 \% \mathrm{CO}_{2}$, apresentou contagem microbiológica até os 21 dias em níveis aceitáveis para consumo $\left(10^{7} \mathrm{UFC} / \mathrm{g}\right)$. A maior quantidade de dióxido de carbono, em conjunto com a baixa temperatura, retardou o desenvolvimento dos microrganismos alvo, havendo sinergismo entre estes dois fatores (FERNANDES ET AL., 2014; BLICKSTAD \& MOLIN, 1983). 


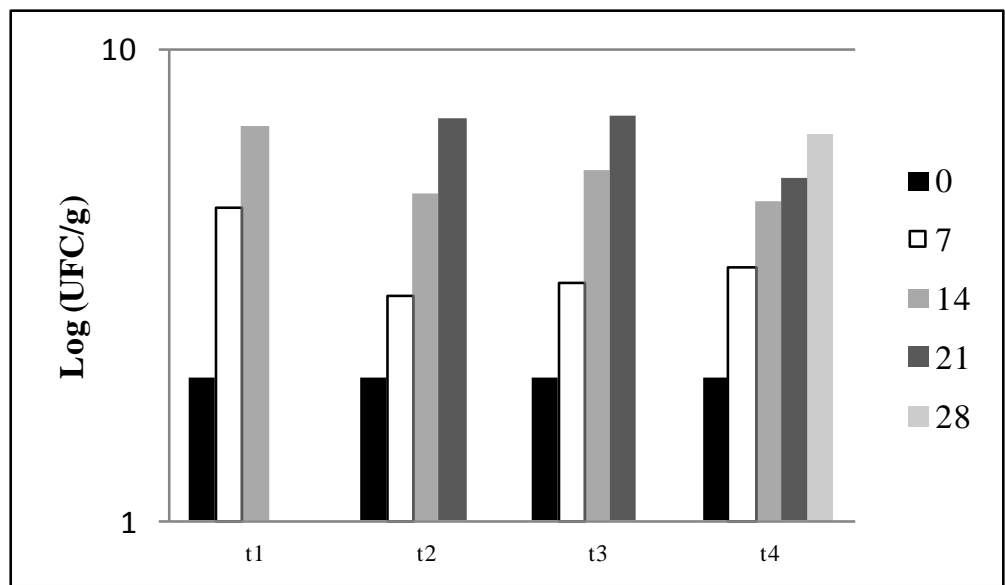

Figura 2. Contagem de microrganismos psicrófilos em amostras acondicionadas sob controle $\left(\mathrm{t}_{1}\right)$, vácuo $\left(\mathrm{t}_{2}\right), 70 \%$ de $\mathrm{CO}_{2}+30 \%$ de $\mathrm{N}_{2}\left(\mathrm{t}_{3}\right)$ e $100 \%$ de $\mathrm{CO}_{2}\left(\mathrm{t}_{4}\right)$ maturadas durante 28 dias a $3^{\circ} \mathrm{C}$.

Segundo Mano et al. (2002), o uso da refrigeração e acondicionamento em AM, além de impedir o crescimento de determinados microrganismos, prolongam as fases de latência e os tempos de duplicação da microbiota que pode desenvolver-se nestas condições. Desta forma, os sistemas de acondicionamento apresentaram diferenças quanto à vida de prateleira dos produtos ao longo do tempo de estocagem e as atmosferas modificadas prolongaram a vida útil em no mínimo 21 dias.

\section{CONCLUSÃO}

Das técnicas de acondicionamento para carne de cordeiro empregadas no presente estudo, o uso de $100 \%$ de $\mathrm{CO}_{2}$, mostrou ser a atmosfera mais indicada, por não apresentar mudanças significativas quantos aos parâmetros de cor, pH, aw e CRA, além da menor contagem microbiana das carnes. Esses resultados corroboram para um uso promissor para o acondicionamento de cortes de pernil ovino sob atmosfera modificada e temperatura de refrigeração.

\section{REFERÊNCIAS}

ABUlARACH, M. L. S; ROCHA, C. E.; FELÍCIO, P. E. Características de Qualidade do Contrafilé (m. L. dorsi) de Touros Jovens da Raça Nelore. Ciênc. Tecnol. Aliment, v. 18, n.2, p.205-210, 1998.

AMERICAN MEAT SCIENCE ASSOCIATION (AMSA). Reasearch guidelines for cookery, sensory and instrumental tenderness measurement of fresh meat. Chicago, 1995. 
ASSOCIATION OF OFFICIAL ANALYTICAL CHEMISTS (AOAC). Official Methods of Analysis. Washington, 2000. 1018p.

BÓRNEZ, R.; LINARES, M. B., \& VERGARA, H. (2010). Effect of different gas stunningmethods on Manchega suckling lamb meat packed under different modified atmospheres. Meat Science, 84(4), 727-734.

DESMOND, E. M.; KENNY, T. A.; WARD, P.; SUN, D-W. Effedt of rapid and conventional cooling et al. 2000

FERNANDES, R. P. P.; FREIRE, M, T, A; PAULA, E. S. M.; KANASHIRO, A. L. S.; CATUNDA, F. A. P; ROSA, A.F; BALIEIRO, J. C. C; TRINDADE, M. A. Stability of lamb loin stored under refrigeration and packed in different modified atmosphere packaging systems. Meat Science 96 (2014) 554-561.

FERNANDES, R. P. P; FREIRE, GUERRA, C.C; CARRER, C.C; BALIEIRO, J. C. C; TRINDADE, M. A. Estabilidade físico-química, microbiológica e sensorial de carne ovina embalada a vácuo estocada sob refrigeração. Ciência Rural, Santa Maria, v.42, n.4, p.724729, abr,2012.

INSTITUTO ADOLFO LUTZ - IAL. Normas Analíticas do Instituto Adolfo Lutz. v. 1:Métodos químicos e físicos para análise de alimentos. 4. ed. São Paulo, 2009.

JAKOBSEN, M.; BERTELSEN, G. Predicting the amount of carbon dioxide absorbed in meat. Meat Science. V. 68, p. 603-610. 2004.

JARDIM, R. D; OSÓRIO, J. C. S; OSÓRIO, M. T. M; MENDONÇA, G; DEL PINO, F. A. B; OLIVEIRA, M; PREDIÉE, G.. Composição Tecidual e Química da Paleta e da Perna em Ovinos da Raça Corriedale. Revista Brasileira Agrociência, Pelotas, v. 13, n. 2, p. 231-236 , abr-jun, 2007.

LAWRIE, R. A \& LEDWARD, D. A. (2006). Lawrie's Meat Science (7th ed.)Cambridge: CRC Press LLC.

LINARES, M. B; BÓRNEZ, R. \& VERGARA, H. (2008). Effect of stunning systems on meat quality of Manchego suckling lamb packed under modified atmospheres. Meat Science, 78(3), 279-287.

MADRUGA, M.S. Qualidade da carne de caprinos e ovinos nativos do Nordeste do Brasil. In: In: SIMPÓSIO INTERNACIONAL DE CONSERVAÇÃO DE RECURSOS GENÉTICOS, 2., 2005, Recife. Anais... Recife: UFRPE, 2005. 1 CD-ROM.

MANO, S.B; PEREDA, J.A.O; FERNANDO, G. D. G. Aumento da vida útil e microbiologia da carne suína embalada em atmosfera modificada. Ciênc. Tecnol. Aliment., Campinas, 22(1): 1-10, jan.-abr. 2002.

MONTE, A. L. S.; GONÇALVES, H. R. O.; VILLARROEL, A. B. S.; DAMACENO, M. N.; CAVALCANTE, A. B. D. Qualidade da carne de caprinos e ovinos: uma revisão. Agropecuária Científica no Semi-Árido, v.8, n.3, p11-17, jul - set , 2012

TERLOUW, C. (2005). Stress reactions at slaughter and meat quality in pigs: Genetic background and prior experience: A brief review of recent findings. Livestock Production Science, 94(1/2), 125-135.

VERGARA, H. \& GALLEGO, L. (2001). Effects of gas composition in modified atmosphere packaging on the meat quality of Spanish Manchega lamb. Journal of the Science of Food and Agriculture, 81(14), 1353-1357. 